\title{
Choroidal excavation with polypoidal choroidal vasculopathy: a case report
}

This article was published in the following Dove Press journal:

Clinical Ophthalmology

24 August 2012

Number of times this article has been viewed

\author{
Wataru Kobayashi' \\ Toshiaki Abe ${ }^{2}$ \\ Hiroshi Tamai' \\ Toru Nakazawa' \\ 'Department of Ophthalmology, \\ 2Division of Clinical Cell Therapy, \\ Center for Advanced Medical \\ Research and Development (ART), \\ Tohoku University Graduate School \\ of Medical Science, Sendai, Japan
}

Correspondence: Wataru Kobayashi Department of Ophthalmology, Tohoku University Graduate School of Medicine, I-I Seiryo-cho, Aoba-ku, Sendai-shi, Miyagi-ken 980-8574, Japan

Tel +8I 227177294

Fax +8I 227177298

Email wkobayashi@oph.med.tohoku.ac.jp
Purpose: This is a report of a case of choroidal excavation accompanied by polypoidal choroidal vasculopathy (PCV) and retinal pigment epithelium detachment (PED).

Methods: A 57-year-old Japanese woman who had begun complaining of metamorphopsia in her left eye 7 months earlier underwent spectral-domain optical coherence tomography (SD-OCT), fluorescein angiography (FA), and indocyanine green angiography (IA), as well as a routine ophthalmological examination.

Results: The patient's intraocular pressure, visual acuity, and visual field were within normal range. Ophthalmoscopy revealed a serous macular detachment, soft drusen, exudates, and a reddish-orange elevated lesion in the macula of the left eye. The right eye was normal. SD-OCT revealed two lesions in the left eye. One was a PED accompanied by a notch sign, and the other was a choroidal excavation. Additionally, FA revealed a window defect in the PED, and IA showed typical PCV. Three monthly injections of antivascular endothelial growth factor preserved visual acuity, but failed to have any visible effect on the lesion during the 6-month follow up period.

Conclusions: This is the first report of choroidal excavation accompanied by PED and PCV. The data suggest that choroidal excavation may be associated with various changes that have not been previously reported. Careful observation of such cases may therefore be necessary.

Keywords: choroidal excavation, polypoidal choroidal vasculopathy, anti-vascular endothelial growth factor treatment

\section{Introduction}

Choroidal excavation, an unusual structural change in the eye, has only recently been discovered thanks to advances in ocular imaging technology and the development of optical coherence tomography (OCT). The first report of choroidal excavations in the macula was made by Jampol et al in 2006, ${ }^{1}$ who used time-domain (TD)-OCT. In $2010,{ }^{2}$ a report was published describing three more cases of choroidal excavation identified using spectral-domain (SD)-OCT, an imaging technique that enables a more detailed description of the morphology of choroidal excavation than TD-OCT. ${ }^{3}$ In 2011, a series of 12 cases of choroidal excavation was documented, ${ }^{4}$ including a case involving a young Japanese patient. ${ }^{5}$ Choroidal excavation as a concept has thus come to be well defined.

The etiology of choroidal excavation, however, remains unclear, partly because the accepted belief has been that this type of lesion is stable and shows little change over time. A report by Wakabayashi et al, for instance, showed three cases of choroidal excavation with stable visual acuity over 6 months. ${ }^{2}$ This stability seemed to indicate 
that choroidal excavation was simply the result of structural changes over a patient's lifetime. However, after finding a case of choroidal excavation accompanied by polypoidal choroidal vasculopathy (PCV), it was believed that choroidal excavation might become a platform for age-related macular degeneration (AMD) or an ischemic lesion such as choroidal neovascularization (CNV).

In this study, we report on the aforementioned case, in which the patient's choroidal excavation was accompanied by PCV and retinal pigment epithelium (RPE) detachment (PED). The expansion of this lesion led to a decrease in visual acuity during the follow-up period. As far as we know, this is the first report of a patient with choroidal excavation and PCV, so we will also discuss the possibility of ischemic change, as well as the treatments we attempted to administer.

\section{Case report}

A 57-year-old woman became aware of metamorphopsia in her left eye over a period of 7 months. The distortion gradually worsened, and she consulted a local ophthalmologist who found an abnormal macular lesion and referred her to our hospital. Her general family medical history and her personal medical history, including her history of ocular disease, were unremarkable.

Her best-corrected visual acuity (BCVA) was $\left(1.2 \times \mathrm{S}-1.5 \mathrm{D}\right.$, cyl-0.75 $\left.\mathrm{D}, \mathrm{Ax} 80^{\circ}\right)$ for the right eye and $\left(1.0 \times \mathrm{S}-2.0 \mathrm{D}\right.$, cyl-0.75 D, Ax90 $\left.{ }^{\circ}\right)$ for the left eye at the time of her first visit. The intraocular pressure was $12 \mathrm{mmHg}$ for both eyes. The anterior segment was normal in both eyes. The Humphrey Field Analyzer (HFA), with a 30-2 Swedish interactive threshold algorithm strategy (Carl Zeiss Meditec Inc, Dublin, CA), did not show any defects in either eye. The cup to disc ratio was 0.7 for the right eye and 0.8 for the left eye. The left fundus showed a small reddish-orange elevated lesion, drusen, exudates, and serous macular detachment in the macular area (Figure 1A). The right fundus showed nothing abnormal (Figure 1B).

Spectral-domain optical coherence tomography (SD-OCT) (Cirrus HD-OCT 4000; Carl Zeiss Meditec Inc) revealed PED with a notch sign, serous retinal detachment, and a choroidal excavation in the macular area of the left eye. The choroidal excavation, which was mainly located in the macula, continued into the PED. SD-OCT further revealed separation between the neural retina, the RPE, and the underlying layers (Figure 1C).

Conditions between the inner segment/outer segment (IS/OS) line and the nerve fiber layer seemed to be almost normal. However, there was a thinned RPE reflective line
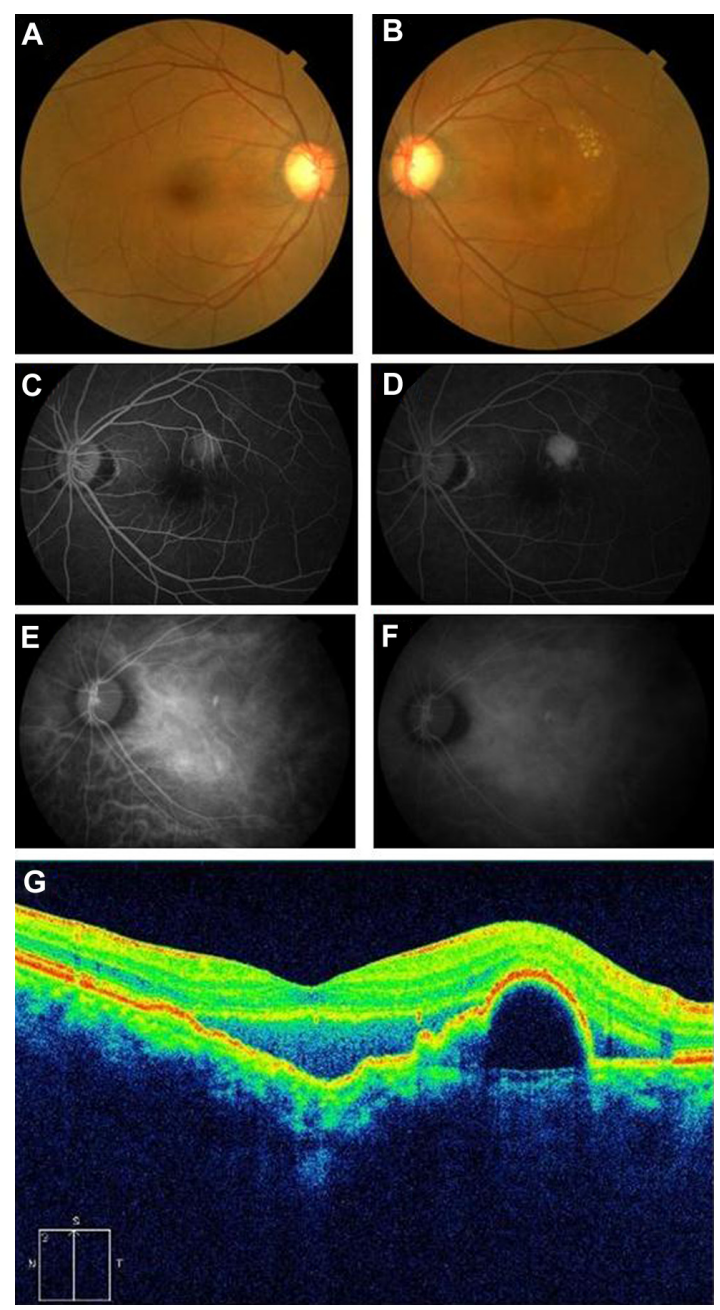

Figure I (A and B) show color fundus photographs of the right and left eyes, respectively. The left eye shows serous retinal detachment and exudates. (C and D) show the results of FA (early and late phase, respectively) and show hyperfluorescence (window defect) at the lesion of the PED. ( $E$ and $\mathbf{F}$ ) show the results of IA (early and late phase, respectively) and show polypoidal fluorescence at the yellowish protruding lesion. SD-OCT demonstrates the separation of the retina between the inner segment and the outer segment junctions of the photoreceptor (IS/OS) line and the RPE with choroidal excavation (G).

Note: A protruding lesion was observed in the upper macular area, and PED ran along the upper part.

Abbreviations: FA, fluorescein angiography; PED, pigment epithelium detachment; SD-OCT, spectral-domain optical coherence tomography; IS/OS, inner segment/ outer segment; IA, indocyanine green augiography; RPE, retinal pigment epithelium.

at the lesion, and we detected a notch sign, a constriction at the lower edge of the PED. We did not detect a doublelayer sign, a highly reflective line seen between the RPE and the choroidal capillary layer. A highly-reflective lump was detected above the serous retinal detachment.

Fluorescein angiography (FA) (Figure 1D and E) revealed a window defect in the area of the PED, and indocyanine green angiography (IA) showed polypoidal hyperfluorescence in the early (Figure 1F) and late phases (Figure 1G). This lesion was coincident with the notch sign detected by OCT. The choroidal excavation lesion appeared unremarkable. 
During successive follow up visits, the serous retinal detachment and exudates increased, and visual acuity gradually decreased. The patient received monthly intravitreal injections of antivascular endothelial growth factor (VEGF) (ranibizumab; $0.5 \mathrm{mg} / 0.05 \mathrm{ml}$ ) to treat the lesions for 3 months, but they did not react well during the first 2 months. Specifically, the serous retinal detachment and exudates increased (Figure 2D-F). However, improvement of the serous retinal detachment was observed after the third injection (Figure $2 \mathrm{H}$ and $\mathrm{I}$ ), and her visual acuity remained at $(0.8)$ at a follow-up visit 6 months later.

\section{Discussion}

The current study is a report on the case of a choroidal excavation accompanied by PCV and PED in the macula. Before this study, fewer than 20 cases of choroidal excavation were documented, and it was believed that the natural course of the disease was stable. Our discovery of a rare case of choroidal excavation associated with PCV contradicts this belief. Such a lesion may be resistant to anti-VEGF therapy, suggesting the importance of noting complications during the longitudinal follow-up period for patients with choroidal excavation.

The structural features of choroidal excavation have only recently been identified thanks to the improvements in image quality provided by OCT. Following the discovery that OCT could be used to detect the tissue microstructure of the human retina and coronary artery, ${ }^{6}$ Fercher et al presented the first in vivo OCT images in $1993 .^{7}$ Many researchers now take advantage of the ever-progressing quality of the acquired images and of the software used to render those images. TD-OCT, an older technology, shows only unclear images of the relative position of the RPE in an optically clear space, ${ }^{1}$ but SD-OCT can provide a clear image of a choroidal excavation. In our case, we used SD-OCT and were able to see the ELM and the IS/OS line very clearly.

In this case, the patient's BCVA was good, but she complained of metamorphopsia. We found a choroidal excavation comprising the layer from the RPE to the underlying choroid,
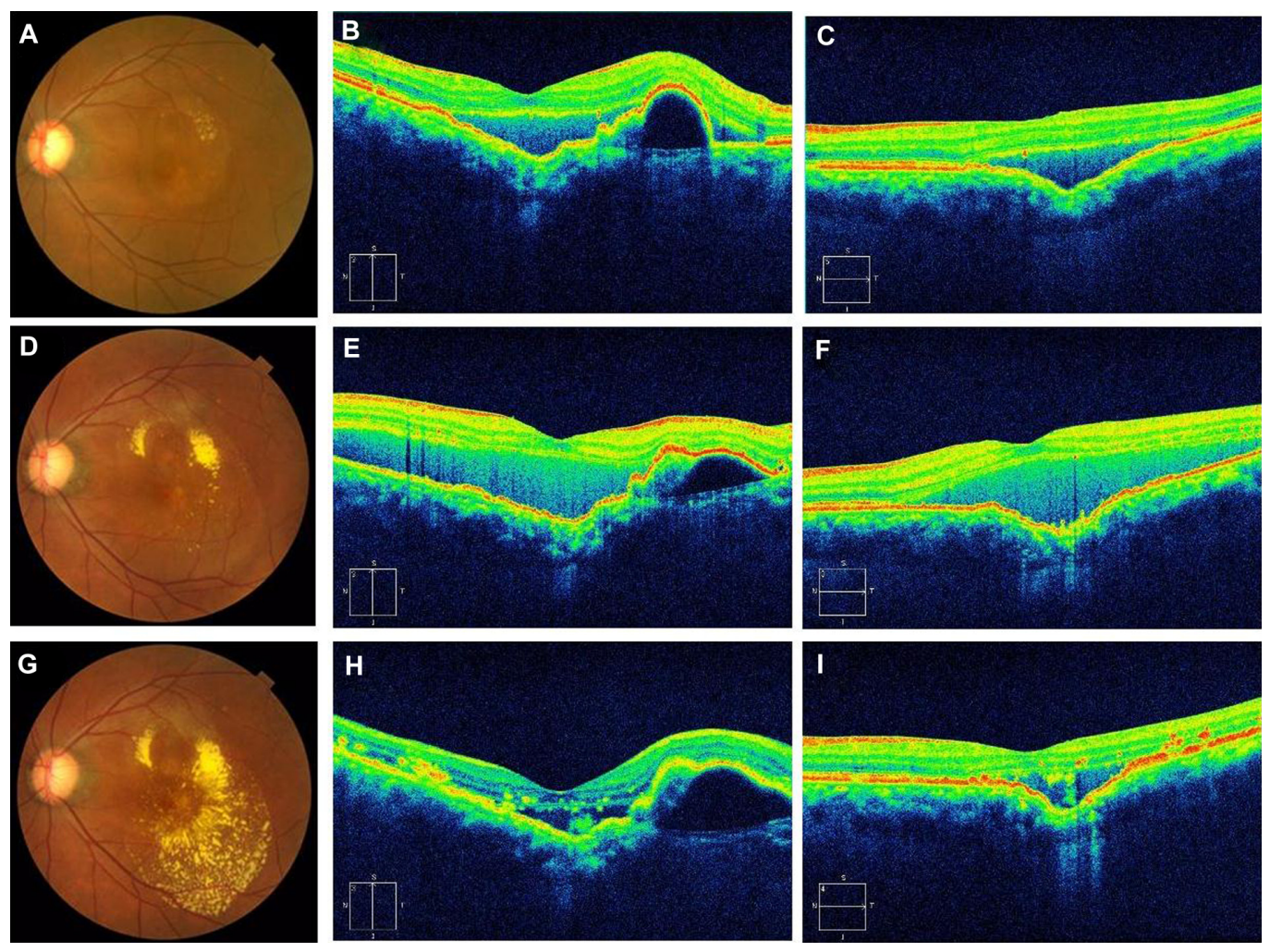

Figure 2 (A-C) are fundus photographs and OCT images before anti-VEGF treatment. (D-F) are fundus photographs and OCT images after initial anti-VEGF treatment. (G-I) are fundus photographs and OCT images after three rounds of anti-VEGF treatment. (B, $\mathbf{E}$ and $\mathbf{H}$ ) are vertical OCT images and (C, F and $\mathbf{I})$ are horizontal OCT images. The left eye shows serous retinal detachment and exudates (A). A choroidal excavation is located just inside the macular area. As described in Figure I, the OCT images show the separation of the retina between the IS/OS line and the RPE (B). The horizontal image shows choroidal excavation more clearly than the vertical image (C). The left eye shows more exudates than that of her initial visit to us (A) (D) and an increase in serous retinal detachment (E and $\mathbf{F})$. The retinal exudates increased after the 3 injections $(\mathbf{G})$, but serous retinal detachment decreased $(\mathbf{H}$ and $\mathbf{I})$.

Note: The extent of PED may have increased.

Abbreviations: OCT, optical coherence tomography; VEGF, vascular endothelial growth factor; IS/OS, inner segment/outer segment; RPE, retinal pigment epithelium; PED, pigment epithelium detachment. 
including separation of the neural retina from the underlying RPE and choroid and localized to the area of the macula. The term "focal choroidal excavation" (FCE) may be a more appropriate representation of her condition. ${ }^{2}$ There are two reported types of FCE; one is a conforming and the other a nonconforming lesion in the neural retina extending to the underlying excavated RPE and choroid. In our patient's case, the lesion was nonconforming.

Margolis et al have reported that the conforming type of lesion can become nonconforming when stress between the photoreceptor chips and the RPE gradually increases. ${ }^{4}$ In our patient's case, there were also additional sources of stress, such as PED and the generation of PCV. Our patient also presented a thinned RPE reflective line, matching the report by Margolis. ${ }^{4}$ In our patient, we were able to clearly see the ELM and IS/OS lines. In Margolis's report, these lines were faint. A clear IS/OS line is believed to be important for good visual acuity, so these results may explain the relatively good visual acuity our patient had when she consulted us.

Choroidal excavations can affect the structure of the RPE and the underlying choroidal layers over a lifetime, sometimes stimulating ischemic change followed by $\mathrm{CNV}^{4}$ or $\mathrm{PCV}$. Margolis has also suggested that such lesions might accompany ischemic changes. ${ }^{4}$ Our patient had a choroidal excavation associated with $\mathrm{CNV}$ and $\mathrm{PCV}$, but no ischemic change, causing us to believe that $\mathrm{CNV}$ and PCV arise not only from bad circulation, but also from a collapse of the Bruch membrane caused by a choroidal excavation. The etiological and pathological mechanism of choroidal excavations is not clear, nor is it clear whether they are congenital or acquired. Evidence is accumulating that suggests they may not be restricted unilaterally, as they do not show clear sex or race predilection. PCV following a choroidal excavation may be accidental. However, the possibility remains that FCE can be followed by devastating changes such as $\mathrm{CNV}$ or PCV, so care should be taken over the long term for patients with FCE.

The BCVA of our patient was over (0.8), a prerequisite for treatment. Our first choice for treatment was a ranibizumab intravitreal injection, rather than photodynamic therapy (PDT), as we were following the Japanese guidelines for PDT. ${ }^{8,9}$

Clinical Ophthalmology

\section{Publish your work in this journal}

Clinical Ophthalmology is an international, peer-reviewed journal covering all subspecialties within ophthalmology. Key topics include: Optometry; Visual science; Pharmacology and drug therapy in eye diseases; Basic Sciences; Primary and Secondary eye care; Patient Safety and Quality of Care Improvements. This journal is indexed on

Submit your manuscript here: http://www.dovepress.com/clinical-ophthalmology-journal
Although the patient's visual acuity remained stable, a transient increase in serous retinal detachment and an increase in retinal exudates suggested it would be difficult to treat these kinds of lesions with anti-VEGF therapy. Had her BCVA declined, we would have been forced to revise her treatment to either PDT alone, or a combination of anti-VEGF therapy and PDT. Ischemic lesions accompanied by FCE may have a more complicated mechanism than that previously reported with respect to $\mathrm{CNV}$ and $\mathrm{PCV}$. Although we are only presenting a case report, we might suggest that more attention should be paid to therapies for lesions in patients with FCE.

In conclusion, the value of this case report is that it presents information on the first known choroidal excavation associated with PCV. Our experience shows that choroidal excavations might be associated with different variable changes than have been previously reported. We believe that choroidal excavation may create circumstances leading to the generation of neovascularization and that there may be a need to reconsider the treatment of lesions associated with FCE in the future.

\section{Disclosure}

The authors report no conflicts of interest in this work.

\section{References}

1. Jampol L, Shankle J, Schroeder R, Tornambe P, Spade RF, Hee MR. Diagnostic and therapeutic challenges. Retina. 2006;26:1072-1076.

2. Wakabayashi Y, Nishimura A, Higashide T, Ijiri S, Sugiyama K. Unilateral choroidal excavation in the macula detected by spectral-domain optical coherence tomography. Acta Ophthalmol. 2010;88(3):e87-e91.

3. Schuman JS. Spectral domain optical coherence tomography for glaucoma (an AOS thesis). Trans Am Ophthalmol Soc. 2008;106:426-458.

4. Margolis R, Mukkamala SK, Jampol LM, et al. The expanded spectrum of focal choroidal excavation. Arch Ophthalmol. 2011;129(10): $1320-1325$.

5. Abe S, Yamamoto T, Kirii E, Yamashita H. Cup-shaped choroidal excavation detected by optical coherence tomography: a case report. Retin Cases Brief Rep. 2010;4(4):373-376.

6. Huang D, Swanson EA, Lin CP, et al. Optical coherence tomography. Science. 1991;254(5035):1178-1181.

7. Fercher AF, Hitzenberger CK, Drexler W, Kamp G, Sattmann H. In vivo optical coherence tomography. Am J Ophthalmol. 1993;116(1): $113-114$.

8. Tano Y. Guidelines for PDT in Japan. Ophthalmology. 2008;115(3): 585-585. e6.

9. Yuzawa M. Polypoidal choroidal vasculopathy. Nihon Ganka Gakkai Zasshi. 2012;116(3):200-232.

PubMed Central and CAS, and is the official journal of The Society of Clinical Ophthalmology (SCO). The manuscript management system is completely online and includes a very quick and fair peer-review system, which is all easy to use. Visit http://www.dovepress.com/ testimonials.php to read real quotes from published authors. 